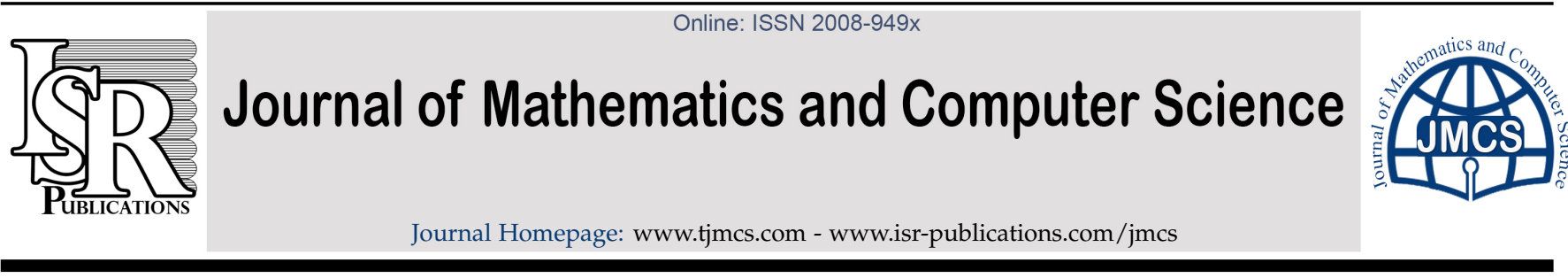

\title{
Bitopological spaces on undirected graphs
}

\author{
Khalid Abdulkalek Abdu ${ }^{\mathrm{a}, \mathrm{b}}$, Adem Kilicman ${ }^{\mathrm{a}, *}$ \\ ${ }^{a}$ Department of Mathematics, University Putra Malaysia (UPM), 4300 Serdang, Selangor, Malaysia. \\ ${ }^{b}$ Department of Accounting, Al-Iraqia University, Adhmia, Baghdad, Iraq.
}

\begin{abstract}
The aim of this article is to associate a bitopological space with every locally finite graph G (a graph in which every vertex is adjacent with finite number of edges). Then some properties of this bitopological space were investigated. After that, connectedness and dense subsets were discussed. Giving a fundamental step toward studying some properties of locally finite graphs by their corresponding bitopological spaces is our motivation.
\end{abstract}

Keywords: Locally finite graph, undirected graphs, bitopological spaces.

2010 MSC: 05C99, 54A05, 54H99.

(C)2018 All rights reserved.

\section{Introduction}

Graph theory is a prominent mathematical tool in many subjects [8] and it is considered as a substantial structure in discrete mathematics for two reasons. First, graphs are mathematically elegant from theoretical viewpoint. Even though graphs are simple relational combinations, they can be used to represent topological spaces, combinatorial objects and many other mathematical combinations. Many concepts will be very useful from practical perspective when they are abstractly represented by graphs and this is the second reason [9]. Topology is an interesting and important field of mathematics because it is a powerful tool that leading to such beneficial concepts as connectivity, continuity, and homotopy. Its influence in most other branches of mathematics is evident [6].

Topologizing discrete structures is a problem that many publications concerned with. One of these discrete structures is graph theory. The investigation of topology on graphs is inspired by the representation of the digital image using a graph model; the points of the image and the connectivity between them are represented by the vertices and the edges of the graph respectively. Therefore, topological properties of the digital images can be studied through topologies on the vertices of graphs [2]. Kelley [7] was the first who formulated the concept of bitopological space, that is, the triple $\left(A, \tau_{1}, \tau_{2}\right)$ of a set $A$ with two

\footnotetext{
${ }^{*}$ Corresponding author

Email addresses: khalid74aso@yahoo.co.uk (Khalid Abdulkalek Abdu), akilicman@yahoo.com (Adem Kilicman)

doi: 10.22436/jmcs.018.02.10
} 
(arbitrary) topologies $\tau_{1}$ and $\tau_{2}$ on A. In 2013, Baby Girija and Pilakkat [1] used the notion of Kelley to study bitopological spaces associated with digraphs $D=(V, E)$ using a nonnegative real valued function $\mathrm{P}$ on $\mathrm{V} \times \mathrm{V}$ called quasi pseudometric that generates tow topologies on $\mathrm{V}$.

The previous work of Baby Girija and Pilakkat of bitopological spaces on graphs was associated with digraphs only. Therefore, our target is to associate a bitopological space with undirected graphs $\mathrm{G}=(\mathrm{V}, \mathrm{E})$ by using two different subbasis families to generate two topologies on $\mathrm{V}$. The first subbasis family introduced by Jafarian Amiri et al. [4] that generates a topology on V, called graphic topology and the other introduced in our previous paper [5] that generate a topology on $V$, called incidence topology. Then, they present a fundamental step toward studying some properties of locally finite graphs by their corresponding bitopological spaces.

In this paper, we associate a bitopological space with every locally finite graph $G=(V, E)$ such that $\mathrm{G}$ is a simple graph. Properties of bitopological space and the relation between this bitopological spaces and corresponding graphs are presented. In Section 2 some definitions of graph theory, topology, and bitopological spaces are shown. In addition, the incidence topology on graphs from our previous paper is introduced. Section 3 is dedicated to main results of bitopological spaces on locally finite graphs. Also this section comprises the connectivity and dense subsets of bitopological space.

\section{Preliminaries}

In this part, some basic notions of graph theory [8,9], topology [6] and bitopological spaces [3, 7] are presented. Furthermore, the incidence topology of simple graphs from our previous paper [5] is introduced.

A (simple) graph $G$ comprises a non-empty collection $V(G)$ of nodes (or vertices), and a collection $E(G)$ of arcs (or edges). Usually $G=(V, E)$ indicates the graph. If $u$ and $v$ are vertices and $e$ is an edge such that $e=u v$, then $u$ and $v$ are adjacent vertices; each vertex $(u$ and $v)$ is incident with $e$. If there are no edges incident with a node $u$, then $u$ is called isolated node. The number of the edges $e \in E$ such that $v$ incident with $e$ is called the degree of the vertex $v$ and denoted by $\mathrm{d}(v)$. A vertex of degree one is called pendent vertex. A graph $\mathrm{G}$ with finite number of nodes and finite number of arcs is called finite graph; otherwise it is an infinite graph. A finite sequence $u_{k} e_{m}, u_{k+1} e_{m+1}, u_{k+2}, \ldots, e_{r} u_{n}$ of distinct vertices and distinct edges, which starts and ends with vertices such that the endpoints of $e_{i}$ are $u_{i-1}$ and $u_{i}$ for each $i$, is called a path and denoted by $P$. The number of edges in a path $P$ is called the length of $P$. If any vertex can be reached from any other vertex in a graph $G$ by traveling along the edges, then $G$ is called connected graph and disconnected otherwise. A connected graph consisting of one vertex adjacent to all others with no cycle is called a star graph and denoted by $S_{n}$.

A topology $\tau$ on a set $A$ is a combination of subsets of $A$, called open, such that the union of the members of any subset of $\tau$ is a member of $\tau$, the intersection of the members of any finite subset of $\tau$ is a member of $\tau$, and both empty set and $A$ are in $\tau$. The ordered pair $(A, \tau)$ is called a topological space. The topology $\tau=P(A)$ on $A$ is called discrete topology while the topology $\tau=\{A, \phi\}$ on $A$ is called trivial (or indiscrete) topology. A topology in which arbitrary intersection of open set is open, called Alexandroff space.

A bitopological space is the triple $\left(A, \tau_{1}, \tau_{2}\right)$ of a collection $A$ with two (arbitrary) topologies $\tau_{1}$ and $\tau_{2}$ on $A$. If $\tau_{1}$ and $\tau_{2}$ are compact, then $\left(A, \tau_{1}, \tau_{2}\right)$ is called double compact. An $(i, j)$-dense subset in $A$ is a subset $B$ of a bitopological space $\left(A, \tau_{1}, \tau_{2}\right)$ such that $\tau_{i} \operatorname{cl}\left(\tau_{j} \operatorname{cl}(B)\right)=A$ and $i, j=1,2$. Also if $\tau_{i} \operatorname{Int}\left(\tau_{j} \mathrm{cl}(B)\right)=\phi$ where $(i, j=1,2)$, i.e., $\tau_{j} \mathrm{cl}(B)$ contains no non-empty $i$-open set, then $B$ is $(i, j)$-nowhere dense subset in $A$. If for each pair of distinct points there exists a $\tau_{1}$-open set or $\tau_{2}$-open set containing one but not the other, then $\left(A, \tau_{1}, \tau_{2}\right)$ is weakly pairwise $T_{0}$. The bitopological space $\left(A, \tau_{1}, \tau_{2}\right)$ is pairwise $\mathrm{T}_{0}$ if for each pair $(a, b)$ of distinct points of $A$, there is either a $\tau_{1}$-open set containing a but not $b$ or there exists a $\tau_{2}$-open set containing $b$ but not $a$. If for each pair of distinct points $a, b$, there exist $a \tau_{1}$-open set $\mathrm{D}$ and $\mathrm{a} \tau_{2}$-open set $W$ such that $\mathrm{a} \in \mathrm{D}, \mathrm{b} \notin \mathrm{D}$ and $\mathrm{b} \in \mathrm{W}, \mathrm{a} \notin W$ or $\mathrm{a} \in \mathrm{W}, \mathrm{b} \notin W$ and $\mathrm{b} \in \mathrm{D}, \mathrm{a} \notin \mathrm{D}$, then $\left(A, \tau_{1}, \tau_{2}\right)$ is weakly pairwise $T_{1}$. The bitopological space $\left(A, \tau_{1}, \tau_{2}\right)$ is weakly pairwise $T_{2}$ if for each 
pair of distinct points $a, b$, there exist a $\tau_{1}$-open set $D$ and a $\tau_{2}$-open set $W$ with $D \cap W=\phi$ such that $a \in D$ and $b \in W$ or $a \in W$ and $b \in D$. If for each pair of distinct points $a, b$, there exist a $\tau_{1}$-open set $\mathrm{D}$ and $a \tau_{2}$-open set $W$ with $\mathrm{D} \cap W=\phi$ such that $a \in D$ and $b \in W$, then $\left(A, \tau_{1}, \tau_{2}\right)$ is pairwise $T_{2}$. If $A$ cannot be expressed as a union of two non-empty open disjoint sets $U$ and $M$ such that $U \in \tau_{1}$ and $M$ $\in \tau_{2}$, then $\left(A, \tau_{1}, \tau_{2}\right)$ is pairwise connected.

Now, we are going to introduce from our previous paper [5] the incidence topology on the set of vertices $V$ of a simple graph $G=(V, E)$ without isolated vertex. Let $I_{e}$ be the incidence vertices with the edge $e$. Define $S_{I G}$ as follows: $S_{I G}=\left\{I_{e} / e \in E\right\}$. Since there is no isolated vertex in $G$, we have $V=\bigcup_{e \in E} I_{e}$. Hence $S_{I G}$ forms a subbasis for a topology $\tau_{I G}$ on $V$, called incidence topology of $G$.

It is obvious that the incidence topologies of the cycle $C_{n} ; n \geqslant 3$, the complete graph $K_{n} ; n \geqslant 3$, and the complete bipartite graph $K_{n, m} ; n, m>1$ are discrete, but the incidence topology of the path $P_{n}$ is not discrete because $P_{n}$ contains two vertices incident with one edge is not open.

Proposition 2.1. Suppose that $\tau_{\mathrm{IG}}$ is the incidence topology of the graph $\mathrm{G}=(\mathrm{V}, \mathrm{E})$. If $\mathrm{d}(v) \geqslant 2$, then $\{v\} \in \tau_{\mathrm{IG}}$ for every $\nu \in \mathrm{V}$.

Proof. Since $\mathrm{G}$ is a simple graph and for any degree of $v$, we have $\bigcap_{i=2}^{\infty} \mathrm{I}_{e_{i}}=\{v\}$ such that $v \in \mathrm{I}_{e_{i}}$ for all $i=2,3, \ldots$. Now by definition of $\tau_{\mathrm{IG}},\{v\}$ is an element in the basis of $\tau_{\mathrm{IG}}$. Hence $\{v\} \in \tau_{\mathrm{IG}}$.

The following corollary is a trivial result for Proposition 2.1.

Corollary 2.2. Let $\mathrm{G}=(\mathrm{V}, \mathrm{E})$ be a graph. If $\mathrm{d}(\mathrm{v}) \geqslant 2$ for all $v \in \mathrm{V}$, then $\tau_{\mathrm{IG}}$ is a discrete topology.

Remark 2.3. Let $\mathrm{G}=(\mathrm{V}, \mathrm{E})$ be a graph, then $\mathrm{I}_{v}$ is the set of all edges incident with the vertex $v$.

Proposition 2.4. In any graph $\mathrm{G}=(\mathrm{V}, \mathrm{E}), \mathrm{U}_{v}=\bigcap_{\mathrm{e} \in \mathrm{I}_{v}} \mathrm{I}_{\mathrm{e}}$ for every $v \in \mathrm{V}$.

Proof. Since $\mathrm{S}_{\mathrm{IG}}$ is the subbasis of $\tau_{\mathrm{IG}}$ and $\mathrm{U}_{v}$ is the intersection of all open set containing $v$, we have $\mathrm{U}_{v}=\bigcap_{e \in \mathrm{A}} \mathrm{I}_{e}$ for some subset $A$ of $\mathrm{E}$. This leads to $v \in \mathrm{I}_{e}$ for each $e \in A$. Therefore, $e \in \mathrm{I}_{v}$ for all $e \in A$. Hence $A \subseteq \mathrm{I}_{v}$ and so $v \in \bigcap_{e \in \mathrm{I}_{v}} \mathrm{I}_{e} \subseteq \mathrm{U}_{v}$. From the definition of $\mathrm{U}_{v}$ the proof is complete.

Corollary 2.5. For any $\mathrm{u}, v \in \mathrm{V}$ in a graph $\mathrm{G}=(\mathrm{V}, \mathrm{E})$, we have $\mathrm{u} \in \mathrm{U}_{v}$ if and only if $\mathrm{I}_{v} \subseteq \mathrm{I}_{\mathrm{u}}$. Equivalently $\mathrm{U}_{v}=\left\{\mathrm{u} \in \mathrm{V} \mid \mathrm{I}_{\mathrm{v}} \subseteq \mathrm{I}_{\mathrm{u}}\right\}$.

Proof. By Proposition 2.4, $\mathrm{U}_{v}=\bigcap_{e \in \mathrm{I}_{v}} \mathrm{I}_{e}$. Therefore, $\mathrm{u} \in \mathrm{U}_{v} \Leftrightarrow u \in \bigcap_{e \in \mathrm{I}_{v}} \mathrm{I}_{e} \Leftrightarrow u$ incident with $e$ for all $\mathrm{e} \in \mathrm{I}_{v} \Leftrightarrow e \in \mathrm{I}_{\mathfrak{u}}$ for all $e \in \mathrm{I}_{v} \Leftrightarrow \mathrm{I}_{v} \subseteq \mathrm{I}_{\mathfrak{u}}$.

Remark 2.6. The Alexandroff topological space $(X, \tau)$ is $T_{1} \Leftrightarrow U_{x}=\{x\}$. It follows that $(X, \tau)$ is discrete. Therefore, the incidence topology $\left(\mathrm{V}, \tau_{\mathrm{IG}}\right)$ which is an Alexandroff space is $\mathrm{T}_{1}$ if and only if it is discrete. Now, if $\left(V, \tau_{I G}\right)$ is an Alexandroff space, then $\left(V, \tau_{I G}\right)$ is $T_{0}$ space if and only if $u_{u}=u_{v}$ implies $u=v$. This means $\mathrm{U}_{u} \neq \mathrm{U}_{v}$ for all distinct pairs of vertices $u, v \in V$. Then from Corollary 2.5, the incidence topology is $\mathrm{T}_{0} \Leftrightarrow \mathrm{I}_{\mathfrak{u}} \neq \mathrm{I}_{v}$ for every distinct pair of vertices $\mathrm{u}, v \in V$.

Proposition 2.7. Let $\mathrm{G}=(\mathrm{V}, \mathrm{E})$ be a graph. Then $\left(\mathrm{V}, \tau_{\mathrm{IG}}\right)$ is a compact topological space if and only if $\mathrm{V}$ is finite.

Proof. Let $\left(\mathrm{V}, \tau_{\mathrm{IG}}\right)$ be a compact topological space. By contradiction, assume that $\mathrm{V}$ is infinite. Then $M_{G}=\left\{U_{v} \mid v \in V\right\}$ is an open covering of $\left(V, \tau_{I G}\right)$ which has no finite subcover. Therefore, $\left(V, \tau_{I G}\right)$ is not compact which is a contradiction. For the converse, it follows directly that $\left(\mathrm{V}, \tau_{\mathrm{IG}}\right)$ is compact since there are only finitely many open subsets on finite space.

Proposition 2.8. Let $\tau_{\mathrm{IG}}$ be the incidence topology of the graph $\mathrm{G}=(\mathrm{V}, \mathrm{E})$, then the set $\mathrm{L}=\{v \in \mathrm{V} \mid \mathrm{d}(v)=1\}$ is closed in $\tau_{\mathrm{IG}}$.

Proof. By assumption $\mathrm{L}=\bigcup_{v \in \mathrm{L}}\{v\}$ and so $\overline{\mathrm{L}}=\overline{\bigcup_{v \in \mathrm{L}}\{v\}}=\bigcup_{v \in \mathrm{L}} \overline{\{v\}}$ by properties of closure. Let $u \in \overline{\mathrm{L}}$, then $u \in \overline{\{v\}}$ for some $v \in L_{\text {and }} \mathrm{I}_{\mathfrak{u}} \subseteq \mathrm{I}_{v}$. Since $\mathrm{d}(v)=1$, then $\mathrm{I}_{v}=\{e\}$ such that $e \in E$. Therefore, $\mathrm{d}(\mathrm{u})=1$ because $\mathrm{I}_{\mathfrak{u}} \subseteq \mathrm{I}_{v}$ and so $\mathrm{u} \in \mathrm{L}$. Hence $\overline{\mathrm{L}} \subseteq \mathrm{L}$ and the proof is complete. 
Proposition 2.9. Let $\mathrm{G}=(\mathrm{V}, \mathrm{E})$ be a connected graph with at least one vertex $v \in \mathrm{V}$ such that $\mathrm{d}(\mathrm{v})=1$. Then the set $M=\{v \in \mathrm{V} \mid \mathrm{d}(v) \geqslant 2)\}$ is dense in $\left(\mathrm{V}, \tau_{\mathrm{IG}}\right)$.

Proof. It is enough to prove that the complement of $M$ has empty interior. For every $v \in M^{c}, v$ is a vertex such that $d(v)=1$. Therefore, $I_{e} \bigcap I_{f} \neq\{v\}$ for every $e, f \in E$, and any two distinct vertices in $M^{c}$ are not adjacent. As a result, for every $\mathrm{B} \subseteq \mathrm{M}^{\mathrm{c}}, \mathrm{B}$ cannot be written as a union of finitely intersection of elements of $S_{I G}$, i.e., $B \notin \tau_{I G}$. Hence $\operatorname{Int}\left(M^{c}\right)=\phi$ and this means $M$ is dense subset in $\left(V, \tau_{I G}\right)$.

All graphs throughout this paper are locally finite simple graphs.

\section{Main results}

\subsection{Bitopological spaces on undirected graphs}

Let $G=(V, E)$ be a simple graph without isolated vertex. Define $S_{G}$ as follows: $S_{G}=\left\{A_{u} \mid u \in V\right\}$ such that $A_{\mathfrak{u}}$ is the set of all vertices adjacent to $u$. Since $G$ has no isolated vertex, we have $V=\bigcup_{\mathfrak{u} \in V} A_{\mathfrak{u}}$. Hence $S_{\mathrm{G}}$ forms a subbasis for a topology $\tau_{\mathrm{G}}$ on $\mathrm{V}$, called graphic topology of $\mathrm{G}$.

Let $I_{e}$ be the incidence vertices with the edge e. Define $S_{I G}$ as follows: $S_{I G}=\left\{I_{e} \mid e \in E\right\}$. Since there is no isolated vertex in $G$, we have $V=\bigcup_{e \in E} I_{e}$. Hence $S_{I G}$ forms a subbasis for a topology $\tau_{I G}$ on $V$, called incidence topology of $\mathrm{G}$.

The above two topologies $\tau_{G}$ and $\tau_{I G}$ on $V$ give the bitopological space $\left(V, \tau_{G}, \tau_{I G}\right)$.

Example 3.1. Let $G=(V, E)$ be a simple graph as in Figure 1 such that $V=\left\{v_{1}, v_{2}, v_{3}, v_{4}\right\}, E=\left\{e_{1}, e_{2}, e_{3}\right\}$. Then $\tau_{\mathrm{G}}=\left\{\phi, V,\left\{v_{2}\right\},\left\{v_{3}\right\},\left\{v_{1}, v_{3}\right\},\left\{v_{2}, v_{4}\right\},\left\{v_{2}, v_{3}\right\},\left\{v_{1}, v_{2}, v_{3}\right\},\left\{v_{2}, v_{3}, v_{4}\right\}\right\}$ and $\tau_{\mathrm{IG}}=\left\{\phi, V,\left\{v_{2}\right\},\left\{v_{3}\right\},\left\{v_{1}, v_{2}\right\}\right.$, $\left.\left\{v_{2}, v_{3}\right\},\left\{v_{3}, v_{4}\right\},\left\{v_{1}, v_{2}, v_{3}\right\},\left\{v_{2}, v_{3}, v_{4}\right\}\right\}$. Therefore, $\tau_{\mathrm{G}}$ and $\tau_{\mathrm{IG}}$ on $\mathrm{V}$ give the bitopological space $\left(\mathrm{V}, \tau_{\mathrm{G}}, \tau_{\mathrm{IG}}\right)$ and clearly that $\tau_{\mathrm{G}}$ and $\tau_{\mathrm{IG}}$ are non-similar.

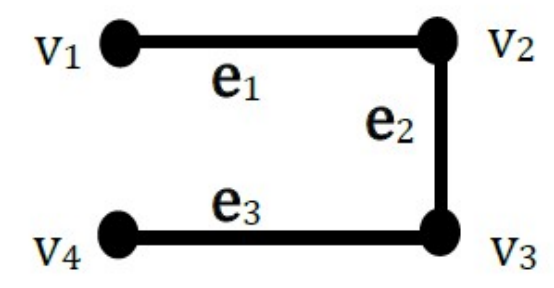

Figure 1: Simple graph with four vertices and three edges.

It is easy to see that, $\tau_{G}$ and $\tau_{I G}$ are non-similar, but they produce the same topology on the ground set $V$ of a graph $G=(V, E)$ when they are both discrete topologies as in the graphs $C_{n}$ and $K_{n} ; n \geqslant 3$.

Remark 3.2. From [4] the graphic topology $\tau_{\mathrm{G}}$ of a graph $\mathrm{G}=(\mathrm{V}, \mathrm{E})$ is discrete if and only if $A_{\mathrm{u}} \nsubseteq \mathrm{A}_{v}$ and $A_{v} \nsubseteq \subseteq A_{u}$ for every distinct pair of vertices $u, v \in V$, and by Corollary 2.2, the incidence topology $\tau_{\text {IG }}$ of a graph $G=(V, E)$ is discrete if $d(v) \geqslant 2$ for every $v \in V$. Therefore, if a graph $G=(V, E)$ satisfies the stipulations above, then $\tau_{\mathrm{G}}$ and $\tau_{\mathrm{IG}}$ are identical topologies (both are discrete topologies).

Proposition 3.3. The bitopological space $\left(\mathrm{V}, \tau_{\mathrm{G}}, \tau_{\mathrm{IG}}\right)$ of a graph $\mathrm{G}=(\mathrm{V}, \mathrm{E})$ is weakly pairwise $\mathrm{T}_{0}$.

Proof. By Remark 2.6, the incidence topology $\tau_{I G}$ of a graph $G=(V, E)$ is $T_{0}$ if and only if $I_{u} \neq I_{v}$ for every distinct pair of vertices $u, v \in V$. Now, if $\mathrm{I}_{\mathfrak{u}}=\mathrm{I}_{v}$, then $u$ and $v$ are adjacent vertices of degree one. By the definition of graphic topology, we have $\{u\},\{v\} \in \tau_{\mathrm{G}}$. Therefore, for every pair of distinct points of $V$ there exists a $\tau_{\mathrm{IG}}$-open set or $\tau_{\mathrm{G}}$-open set containing one but not the other. Hence the bitopological space $\left(V, \tau_{G}, \tau_{I G}\right)$ of a graph $G=(V, E)$ is weakly pairwise $T_{0}$. 
Proposition 3.4. The bitopological space $\left(\mathrm{V}, \tau_{\mathrm{G}}, \tau_{\mathrm{IG}}\right)$ of a graph $\mathrm{G}=(\mathrm{V}, \mathrm{E})$ is pairwise $\mathrm{T}_{0}$.

Proof. Let $(u, v)$ be any distinct pair of points of $V$. Then there are two cases.

Case 1: $u$ and $v$ are adjacent vertices. From definition of graphic topology $\tau_{\mathrm{G}}$, there are two $\tau_{\mathrm{G}}-$ open sets $A_{\mathfrak{u}}$ and $A_{v}$ such that $A_{u}$ containing $v$ but not $u$ and $A_{v}$ containing $u$ but not $v$.

Case 2: $u$ and $v$ are not adjacent vertices. This means there exist two different edges $e, f \in E$ such that $u$ incident with $e$ and $v$ incident with $f$. By the definition of incidence topology $\tau_{I G}, I_{e}$ and $I_{f}$ are two $\tau_{\mathrm{IG}}$-open sets such that $\mathrm{I}_{e}$ containing $u$ but not $v$ and $\mathrm{I}_{\mathrm{f}}$ containing $v$ but not $\mathrm{u}$.

From cases above, for each pair $(u, v)$ of distinct points of $V$, there is either a $\tau_{G}$-open set containing $u$ but not $v$ or there exist a $\tau_{\mathrm{IG}}$-open set containing $\nu$ but not $u$. Hence the bitopological space $\left(V, \tau_{\mathrm{G}}, \tau_{\mathrm{IG}}\right)$ is pairwise $T_{0}$.

Proposition 3.5. The bitopological space $\left(\mathrm{V}, \tau_{\mathrm{G}}, \tau_{\mathrm{IG}}\right)$ of a graph $\mathrm{G}=(\mathrm{V}, \mathrm{E})$ is weakly pairwise $\mathrm{T}_{1}$ if and only if $A_{\mathfrak{u}} \neq A_{v}$ and $I_{\mathfrak{u}} \neq I_{v}$ for every distinct pair of vertices $u, v \in V$.

Proof. $(\Rightarrow)$ Let $\left(\mathrm{V}, \tau_{\mathrm{G}}, \tau_{\mathrm{IG}}\right)$ be a weakly pairwise $\mathrm{T}_{1}$ bitopological space. By contradiction, suppose that there exists a distinct pair of vertices $u, v \in V$ such that $A_{\mathfrak{u}}=A_{v}$ or $I_{\mathfrak{u}}=I_{v}$.

(i) If $A_{\mathfrak{u}}=A_{v}$, then from [4], $\tau_{G}$ is not a $T_{0}$ space, i.e., there is no $\tau_{G}$-open set containing $u$ but not $v$ or containing $v$ but not $u$ which is a contradiction with the assumption since $\left(V, \tau_{G}, \tau_{I G}\right)$ is weakly pairwise $\mathrm{T}_{1}$.

(ii) If $I_{\mathfrak{u}}=I_{v}$, then by Remark 2.6, $\tau_{I G}$ is not a $T_{0}$ space, i.e., there is no $\tau_{I G}$-open set containing $u$ but not $v$ or containing $v$ but not $u$ which is a contradiction with the assumption since $\left(V, \tau_{G}, \tau_{I G}\right)$ is weakly pairwise $T_{1}$.

$(\Leftarrow)$ Let $A_{\mathfrak{u}} \neq A_{v}$ and $I_{\mathfrak{u}} \neq I_{v}$ for every distinct pair of vertices $u, v \in V$. For each pair of distinct points $u, v \in V$, we have the following cases:

Case 1: $u$ and $v$ are adjacent vertices. From definition of graphic topology $\tau_{\mathrm{G}}$, there are two $\tau_{\mathrm{G}^{-}}$open sets $A_{u}$ and $A_{v}$ such that $A_{u}$ containing $v$ but not $u$ and $A_{v}$ containing $u$ but not $v$. From assumption $\mathrm{I}_{\mathfrak{u}} \neq \mathrm{I}_{v}$ for every distinct pair of vertices $u, v \in V$. Then by Remark 2.6, $\tau_{\mathrm{IG}}$ is a $\mathrm{T}_{0}$ space, i.e., there exists a $\tau_{\mathrm{IG}}$-open set containing $u$ but not $v$ or containing $v$ but not $u$.

Case 2: $u$ and $v$ are not adjacent vertices. This means there exist two different edges $e, f \in E$ such that $u$ incidents with $e$ and $v$ incidents with $f$. By definition of incidence topology $\tau_{I G}, I_{e}$ and $I_{f}$ are two $\tau_{\mathrm{IG}}$-open sets such that $\mathrm{I}_{e}$ containing $u$ but not $v$ and $\mathrm{I}_{\mathrm{f}}$ containing $v$ but not $u$. From assumption $A_{\mathfrak{u}} \neq A_{v}$ for every distinct pair of vertices $u, v \in V$. Thus $\tau_{G}$ is $T_{0}$ space (see [4]), i.e., there exist a $\tau_{G^{-}}$open set containing $u$ but not $v$ or containing $v$ but not $u$.

From cases above, for each pair of distinct vertices $u, v \in V$, there exists a $\tau_{\mathrm{G}}$ - open set $\mathrm{D}$ and $\tau_{\mathrm{IG}}$ - open set $W$ such that either $u \in D, v \notin D$ and $v \in W, u \notin W$ or $u \in W, v \notin W$ and $v \in D, u \notin D$. Hence the bitopological space $\left(V, \tau_{G}, \tau_{I G}\right)$ is weakly pairwise $T_{1}$.

Proposition 3.6. The bitopological space $\left(\mathrm{V}, \tau_{\mathrm{G}}, \tau_{\mathrm{IG}}\right)$ of a graph $\mathrm{G}=(\mathrm{V}, \mathrm{E})$ is pairwise $\mathrm{T}_{1}$ if and only if $\tau_{\mathrm{G}}$ and $\tau_{\mathrm{IG}}$ are discrete topologies.

Proof. $\left(\mathrm{V}, \tau_{\mathrm{G}}, \tau_{\mathrm{IG}}\right)$ is pairwise $\mathrm{T}_{1}$ bitopological space if and only if each of $\tau_{\mathrm{G}}$ and $\tau_{\mathrm{IG}}$ is $\mathrm{T}_{1}$ space because pairwise $T_{1}$ in bitopological space is equivalent to $T_{1}$ in each topology (see [7]) if and only if $\tau_{G}$ and $\tau_{I G}$ are discrete topologies since from [4] and Remark 2.6, $\tau_{\mathrm{G}}$ and $\tau_{\mathrm{IG}}$ are $\mathrm{T}_{1}$ spaces if and only if $\tau_{\mathrm{G}}$ and $\tau_{\mathrm{IG}}$ are discrete topologies.

Proposition 3.7. The bitopological space $\left(\mathrm{V}, \tau_{\mathrm{G}}, \tau_{\mathrm{IG}}\right)$ of a graph $\mathrm{G}=(\mathrm{V}, \mathrm{E})$ is weakly pairwise $\mathrm{T}_{2}$ if and only if $\mathrm{A}_{\mathfrak{u}} \neq \mathrm{A}_{v} ; \mathrm{I}_{\mathrm{u}} \neq \mathrm{I}_{v}$ for every distinct pair of vertices $\mathrm{u}, v \in \mathrm{V}$; and the length of any path between any two distinct pendent vertices is at least four. 
Proof. $(\Rightarrow)$ Let $\left(\mathrm{V}, \tau_{\mathrm{G}}, \tau_{\mathrm{IG}}\right)$ be a weakly pairwise $\mathrm{T}_{2}$ bitopological space. By contradiction, suppose that there exists a distinct pair of vertices $u, v \in V$ such that $A_{\mathfrak{u}}=A_{v}$ or $I_{\mathfrak{u}}=I_{v}$ or there is a path of length less than four between two distinct pendent vertices.

(i) If $A_{u}=A_{v}$, then from [4], $\tau_{G}$ is not a $T_{0}$ space, i.e., there is no $\tau_{G}$-open set containing $u$ but not $v$ or containing $v$ but not $u$ which is a contradiction with the assumption since $\left(V, \tau_{G}, \tau_{I G}\right)$ is weakly pairwise $\mathrm{T}_{2}$.

(ii) If $I_{\mathfrak{u}}=I_{v}$, then by Remark 2.6, $\tau_{I G}$ is not a $T_{0}$ space, i.e., there is no $\tau_{I G}$-open set containing $u$ but not $v$ or containing $v$ but not $u$ which is a contradiction with the assumption since $\left(V, \tau_{\mathrm{G}}, \tau_{\mathrm{IG}}\right)$ is weakly pairwise $T_{2}$.

(iii) Suppose that $u$ and $v$ are two distinct pendent vertices and $P$ is a path of length less than four between $u$ and $v$. Therefore, the length of $P$ is three since if the length is one or two, then $A_{\mathfrak{u}}=A_{v}$ or $\mathrm{I}_{u}=I_{v}$, respectively which is a contradiction with the assumption. Now, let $\mathrm{P}=\mathrm{ue}_{1} x \mathrm{e}_{2} y e_{3} v$ such that $u, x, y, v \in V$ and $e_{1}, e_{2}, e_{3} \in E$. Then the open sets in $S_{G}$ of $\tau_{G}$ that contain $u$ and $v$ are $A_{x}$ and $A_{y}$ such that $u, y \in A_{x}$ and $x, v \in A_{y}$. Also the open sets in $S_{I G}$ of $\tau_{I G}$ that contain $u$ and $v$ are $I_{e_{1}}=\{u, x\}$ and $\mathrm{I}_{e_{3}}=\{y, v\}$, respectively. Clearly, $A_{x} \cap \mathrm{I}_{e_{3}} \neq \phi$ and $A_{y} \cap \mathrm{I}_{e_{1}} \neq \phi$. Thus, there is no $\tau_{\mathrm{G}}$-open set $\mathrm{D}$ and $\tau_{\mathrm{IG}}$-open set $\mathrm{W}$ such that $u \in \mathrm{D}$ and $v \in W$ or $u \in W$ and $v \in \mathrm{D}$, which is a contradiction with the assumption since $\left(V, \tau_{G}, \tau_{I G}\right)$ is weakly pairwise $T_{2}$.

$(\Leftarrow)$ Assume that $A_{\mathfrak{u}} \neq A_{v} ; I_{\mathfrak{u}} \neq I_{v}$ for every distinct pair of vertices $u, v \in V$; and the length of any path between any two distinct pendent vertices is at least four. For any pair of distinct vertices $u, v \in V$, we have the following cases:

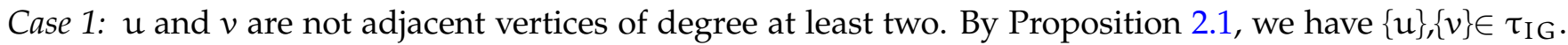
From assumption $A_{u} \neq A_{v}$ for every distinct pair of vertices $u, v \in V$, then $\tau_{G}$ is $T_{0}$ space (see [4]), i.e., there exists a $\tau_{\mathrm{G}}$-open set containing $u$ but not $v$ or containing $v$ but not $u$. Therefore, there exist $\tau_{\mathrm{G}}$-open set $\mathrm{D}$ and $\tau_{\mathrm{IG}}$-open set $\mathrm{W}$ with $\mathrm{D} \cap \mathrm{W}=\phi$ such that $u \in \mathrm{D}$ and $v \in W$ or $u \in W$ and $v \in \mathrm{D}$.

Case 2: $u$ and $v$ are adjacent vertices such that $d(u)=1$ and $d(v) \geqslant 2$. By Proposition 2.1, $\{v\} \in \tau_{\mathrm{IG}}$. From definition of graphic topology, $A_{v}$ is an open set containing $u$ but not $v$. Hence, $A_{v}$ is $\tau_{\mathrm{G}}$-open set containing $u$ and $\{v\}$ is $\tau_{I G}$-open set containing $v$ such that $A_{v} \bigcap\{v\}=\phi$.

Case 3: $u$ and $v$ are not adjacent vertices such that $d(u)=1$ and $d(v) \geqslant 2$. Suppose that $x \in V$ is a vertex adjacent with $u$. This means there exists an edge $e \in E$ such that $e=u x$. Now, either $v$ is adjacent with $x$ or $v$ is not adjacent with $x$.

(a) If $v$ is adjacent with $x$, then $\mathrm{I}_{e}=\{u, x\}$ is $\tau_{\mathrm{IG}}$-open set by the definition of incidence topology. From [4], the smallest open set in $\tau_{G}$ containing $v$ is $u_{v}=\left\{y \in V \mid A_{v} \subseteq A_{y}\right\}$. Since $d(u)=1$ and $d(v) \geqslant 2$, then $A_{v} \nsubseteq A_{u}$. Also, $A_{v} \nsubseteq A_{x}$ since $v$ is adjacent with $x$. As a result, $u, x \notin U_{v}$. Hence, $I_{e}$ is $\tau_{\mathrm{IG}}$-open set containing $\mathrm{u}$ and $\mathrm{U}_{v}$ is $\tau_{\mathrm{G}}$-open set containing $v$ such that $\mathrm{I}_{e} \bigcap \mathrm{U}_{v}=\phi$.

(b) If $v$ is not adjacent with $x$, then $A_{x}$ is an open set containing $u$ but not $v$ by the definition of graphic topology. By Proposition 2.1, $\{v\} \in \tau_{\mathrm{IG}}$. Therefore, $A_{x}$ is $\tau_{\mathrm{G}}$-open set containing $u$ and $\{v\}$ is $\tau_{\mathrm{IG}}$-open set containing $v$ such that $A_{x} \bigcap\{v\}=\phi$.

Case 4: $u$ and $v$ are not adjacent vertices such that $d(u)=d(v)=1$. From assumption, the length of any path between $u$ and $v$ is at least four. Let $\mathrm{P}=u_{1} x e_{2} y e_{3} z e_{4} v$ be a path between $u$ and $v$ such that $u, x, y, z, v \in V$ and $e_{1}, e_{2}, e_{3}, e_{4} \in E$. By definition of graphic topology and incidence topology, $A_{x}$ and $I_{e_{4}}$ are $\tau_{\mathrm{G}}$-open set and $\tau_{\mathrm{IG}}$-open set, respectively, with $A_{x} \cap \mathrm{I}_{e_{4}}=\phi$ such that $u \in A_{x}$ and $v \in \mathrm{I}_{e_{4}}$.

From cases above, for each pair of distinct vertices $u, v \in V$ there exist $\tau_{\mathrm{G}}$-open set $\mathrm{D}$ and $\tau_{\mathrm{IG}}$-open set $W$ with $\mathrm{D} \cap \mathrm{W}=\phi$ such that $u \in \mathrm{D}$ and $v \in W$ or $u \in W$ and $v \in D$. Hence the bitopological space $\left(V, \tau_{G}, \tau_{I G}\right)$ is weakly pairwise $T_{2}$.

Proposition 3.8. Let $\left(\mathrm{V}, \tau_{\mathrm{G}}, \tau_{\mathrm{IG}}\right)$ be the bitopological space of a graph $\mathrm{G}(\mathrm{V}, \mathrm{E})$. Then the two statements below are equivalent. 
(1) $\left(\mathrm{V}, \tau_{\mathrm{G}}, \tau_{\mathrm{IG}}\right)$ is pairwise $\mathrm{T}_{2}$ (pairwise Hausdorff).

(2) $\left(\mathrm{V}, \tau_{\mathrm{G}}, \tau_{\mathrm{IG}}\right)$ is pairwise $\mathrm{T}_{1}$.

Proof. $(1) \Rightarrow(2)$ is evident. Now suppose that $\left(\mathrm{V}, \tau_{\mathrm{G}}, \tau_{\mathrm{IG}}\right)$ is pairwise $\mathrm{T}_{1}$. By Proposition $3.6, \tau_{\mathrm{G}}$ and $\tau_{\mathrm{IG}}$ are discrete topologies. This means each singleton is an open set in $\tau_{\mathrm{G}}$ and $\tau_{\mathrm{IG}}$. Therefore, for each pair of distinct vertices $u, v \in V$ there exist $\tau_{\mathrm{G}}$-open set $\mathrm{D}$ and $\tau_{\mathrm{IG}}$-open set $\mathrm{W}$ such that $u \in \mathrm{D}, v \in W$ and $\mathrm{D} \cap \mathrm{W}=\phi$. Hence, $\left(\mathrm{V}, \tau_{\mathrm{G}}, \tau_{\mathrm{IG}}\right)$ is pairwise $\mathrm{T}_{2}$.

Remark 3.9. From [4] and Proposition 2.7, the topology $\left(\tau_{\mathrm{G}}\right.$ and $\tau_{\mathrm{IG}}$ respectively) of a graph $\mathrm{G}=(\nu, E)$ are compact if and only if $\mathrm{V}$ is finite. Therefore, the bitopological space $\left(\mathrm{V}, \tau_{\mathrm{G}}, \tau_{\mathrm{IG}}\right)$ is double compact if and only if $\mathrm{V}$ is finite.

\subsection{Connectedness in bitopological spaces $\left(\mathrm{V}, \tau_{\mathrm{G}}, \tau_{\mathrm{IG}}\right)$}

The sufficient conditions for connectivity of the bitopological space $\left(V, \tau_{G}, \tau_{I G}\right)$ of a graph $G=(V, E)$ are presented in this section.

Proposition 3.10. The bitopological space $\left(\mathrm{V}, \tau_{\mathrm{G}}, \tau_{\mathrm{IG}}\right)$ of a graph $\mathrm{G}=(\mathrm{V}, \mathrm{E})$ such that $\mathrm{d}(v) \geqslant 2$ for all $v \in \mathrm{V}$ is disconnected.

Proof. If $\mathrm{d}(v) \geqslant 2$ for all $v \in \mathrm{V}$ in a graph $\mathrm{G}=(\mathrm{V}, \mathrm{E})$, then $\tau_{\mathrm{IG}}$ is discrete topology by Corollary 2.2. Also the graphic topology $\tau_{G}$ for any graph $G=(V, E)$ is not an indiscrete topology since $A_{\mathfrak{u}} \neq V$ for all $u \in V$. Therefore, for any open set $A \in \tau_{G}$ there exists an open set $A^{c} \in \tau_{I G}$ such that $V=A \cup A^{c}$. Hence $\left(\mathrm{V}, \tau_{\mathrm{G}}, \tau_{\mathrm{IG}}\right)$ is disconnected bitopological space.

Corollary 3.11. The bitopological space $\left(\mathrm{V}, \tau_{\mathrm{G}}, \tau_{\mathrm{IG}}\right)$ of the complete graph $\mathrm{K}_{n}$, the cycle $\mathrm{C}_{n}$, and the wheel $\mathrm{W}_{\mathrm{n}}$ are disconnected such that $\mathrm{n} \geqslant 3$.

Proof. From definition of $K_{n}, C_{n}$, and $W_{n}, d(v) \geqslant 2$ for all $v \in K_{n}, C_{n}, W_{n}$. Then by Proposition 3.10, $\left(\mathrm{V}, \tau_{\mathrm{G}}, \tau_{\mathrm{IG}}\right)$ is disconnected bitopological space.

Proposition 3.12. The bitopological space $\left(\mathrm{V}, \tau_{\mathrm{G}}, \tau_{\mathrm{IG}}\right)$ of every disconnected graph $\mathrm{G}=(\mathrm{V}, \mathrm{E})$ is disconnected.

Proof. Suppose that $\left\{G_{i}, i \in N\right\}$ is the set of all components (connected subgraphs) of $G$ such that $G_{i}=$ $\left(V_{i}, E_{i}\right)$. For every component $G_{i}, i \in N$ we have $\bigcup_{u \in V} A_{u}=V\left(G_{i}\right)$ such that $A_{\mathfrak{u}}$ is an open set for all $u \in V_{i}$ in $\tau_{G}$ and $\bigcup_{e \in E_{i}} I_{e}=V\left(G_{i}\right)$ such that $I_{e}$ is an open set for all $e \in E_{i}$ in $\tau_{I G}$. As a result, $V\left(G_{i}\right) \in \tau_{G}$ and $\tau_{I G}$. Since $\left[V\left(G_{i}\right)\right]^{c}$ in $V(G)$ is the union of vertices of other components, thus $\left[V\left(G_{i}\right)\right]^{c} \in \tau_{G}$ and $\tau_{I G}$. Then we have $V(G)=V\left(G_{i}\right) \cup\left[V\left(G_{i}\right)\right]^{c}$ for every $i \in N$. Therefore, $V(G)$ is the union of two disjoint sets $V\left(G_{i}\right)$ and $\left[V\left(G_{i}\right)\right]^{c}$ such that $V\left(G_{i}\right) \in \tau_{G} \backslash\{\phi\}$ and $\left[V\left(G_{i}\right)\right]^{c} \in \tau_{I G} \backslash\{\phi\}$ or $V\left(G_{i}\right) \in \tau_{I G} \backslash\{\phi\}$ and $\left[V\left(G_{i}\right)\right]^{c} \in \tau_{G} \backslash\{\phi\}$. Hence the bitopological space $\left(V, \tau_{G}, \tau_{I G}\right)$ is disconnected.

Now, suppose that $G=(V, E)$ is a connected graph. The bitopological space $\left(V, \tau_{G}, \tau_{I G}\right)$ of a graph $\mathrm{G}=(V, E)$ such that $n(V)=2$ which is the star graph $S_{2}$ is connected since $\tau_{I G}$ is indiscrete topology. Also if $\tau_{G}$ and $\tau_{I G}$ are discrete topologies, then the bitopological space $\left(V, \tau_{G}, \tau_{I G}\right)$ is disconnected as in the graphs $C_{n}$ and $K_{n} ; n \geqslant 3$.

Proposition 3.13. The bitopological space $\left(\mathrm{V}, \tau_{\mathrm{G}}, \tau_{\mathrm{IG}}\right)$ of the star graph $\mathrm{S}_{\mathrm{n}}$ such that $\mathrm{n} \geqslant 3$ is disconnected bitopological space.

Proof. From definition of star graph, $\bigcap_{e \in E} I_{e}=\{u\}$ such that $u \in V$. This means $u$ is adjacent with all vertices of the set $V \backslash\{u\}$ and so $d(u) \geqslant 2$. From definition of graphic topology $\tau_{G}, A_{u}$ is an open set such that $v \in A_{\mathfrak{u}}$ for all $v \in V \backslash\{u\}$. By Proposition 2.1, $\{u\} \in \tau_{\text {IG }}$ because $d(u) \geqslant 2$. Thus $V=A_{u} \bigcup\{u\}$ and so $V$ is a union of two non-empty open disjoint sets such that $A_{\mathfrak{u}} \in \tau_{G}$ and $\{u\} \in \tau_{I G}$. Hence $\left(V, \tau_{G}, \tau_{I G}\right)$ is disconnected bitopological space. 
Example 3.14. Consider the star graph $S_{4}$ in Figure 2. The graphic topology of $S_{4}$ is $\tau_{S_{4}}=\left\{\phi, V,\left\{v_{1}\right\},\left\{v_{2}, v_{3}\right.\right.$, $\left.\left.v_{4}\right\}\right\}$ and the incidence topology is $\tau_{\mathrm{IS}}=\left\{\phi, \mathrm{V},\left\{v_{1}\right\},\left\{v_{1}, v_{2}\right\},\left\{v_{1}, v_{3}\right\},\left\{v_{1}, v_{4}\right\},\left\{v_{1}, v_{2}, v_{3}\right\},\left\{v_{1}, v_{2}, v_{4}\right\},\left\{v_{1}, v_{3}, v_{4}\right\}\right\}$. Thus $V=\left\{v_{2}, v_{3}, v_{4}\right\} \bigcup\left\{v_{1}\right\}$ such that $\left\{v_{2}, v_{3}, v_{4}\right\} \in \tau_{S_{4}}$ and $\left\{v_{1}\right\} \in \tau_{\mathrm{IS}}$.

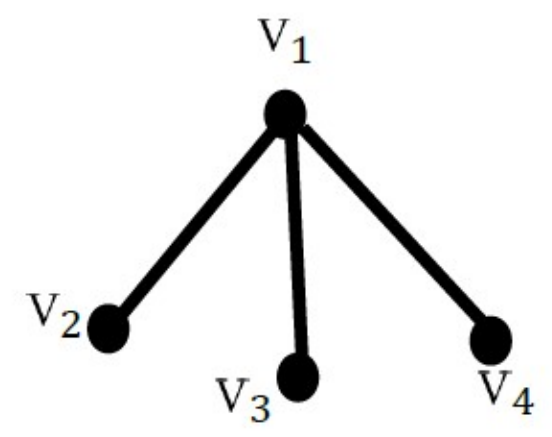

Figure 2: The star graph $S_{4}$.

The next proposition gives the sufficient condition for connectedness of the bitopological space $\left(V, \tau_{G}, \tau_{I G}\right)$ of a connected graph $G=(V, E)$ which is not a star with at least one pendent vertex, i.e., $\tau_{I G}$ is not a discrete topology.

Proposition 3.15. Let $\mathrm{G}=(\mathrm{V}, \mathrm{E})$ be a connected graph which is not a star with at least one pendent vertex. Then the bitopological space $\left(\mathrm{V}, \tau_{\mathrm{G}}, \tau_{\mathrm{IG}}\right)$ is pairwise connected if and only if for every $v \in \mathrm{V}$ such that $\mathrm{d}(v) \geqslant 2, v$ is adjacent with at least one pendent vertex.

Proof. $(\Rightarrow)$ Let $\left(V, \tau_{G}, \tau_{I G}\right)$ be a pairwise connected bitopological space. By contradiction, suppose that there exists a vertex $v \in V$ such that $\mathrm{d}(v) \geqslant 2$ and $v$ is not adjacent with a pendent vertex. By the definition of graphic topology, we have $\bigcap_{\mathfrak{u} \in V} A_{\mathfrak{u}}=\{v\}$ such that $u$ is adjacent with $v$. Therefore, $\{v\} \in \tau_{\mathrm{G}}$ since $\tau_{\mathrm{G}}$ is an Alexandroff space. Since $v$ is not adjacent with a pendent vertex, $\bigcup_{e \in E} I_{e}=V \backslash\{v\}$ such that $e$ is not incident with $v$. By definition of incidence topology, $V \backslash\{v\} \in \tau_{\mathrm{IG}}$. Thus $V=\{v\} \bigcup V \backslash\{v\}$ and so $V$ is a union of two non-empty open disjoint sets such that $\{v\} \in \tau_{\mathrm{G}}$ and $V \backslash\{v\} \in \tau_{\mathrm{IG}}$. Hence $\left(\mathrm{V}, \tau_{\mathrm{G}}, \tau_{\mathrm{IG}}\right)$ is disconnected which is a contradiction with the assumption. Similarly if $G$ has more than one vertex of degree at least two and not adjacent with a pendent vertex, then $\left(V, \tau_{G}, \tau_{I G}\right)$ is disconnected.

$(\Leftarrow)$ Let $M$ be the set of all vertices $v \in V$ such that $\mathrm{d}(v) \geqslant 2$. From assumption, for all $v \in M, v$ is adjacent with at least one pendent vertex. Therefore, $U I_{e} \neq V \backslash P$ for some $e \in E$ and $P \subseteq M$. This means $V \backslash P \notin \tau_{I G}$ such that $P \subseteq M$. From the definition of graphic topology $\tau_{G}$, we have $\{v\} \in \tau_{G}$ for all $v \in M$. Now, for any open set $A \in \tau_{G} \backslash\{\phi, V\}$, we have the following cases:

Case 1: $A \subseteq M$. Then $A^{c} \notin \tau_{I G}$ since $V \backslash P \notin \tau_{I G}$ such that $P \subseteq M$.

Case 2: $A=\bigcup A_{u}$ for some $u \in M$. Then there exists at least one vertex $v \in A$ such that $v \neq u$ and $\mathrm{d}(v) \geqslant 2$ because $\mathrm{G}$ is not a star. If $A^{c} \in \tau_{\mathrm{IG}}$, then $v$ is not adjacent with a pendent vertex which is a contradiction with the assumption.

Case 3: $A=\bigcup\left(A_{\mathfrak{u}} \bigcup\{u\}\right)$ for some $u \in M$. The proof is similar as Case 2 .

From cases above, for all open set $A \in \tau_{G} \backslash\{\phi, V\}, A^{c} \notin \tau_{I G}$. Hence $\left(V, \tau_{G}, \tau_{I G}\right)$ is pairwise connected bitopological space.

\subsection{Density in bitopological spaces $\left(\mathrm{V}, \tau_{\mathrm{G}}, \tau_{\mathrm{IG}}\right)$}

Some necessary conditions for dense subsets and nowhere dense subsets, which their definitions are mentioned in preliminaries, of the bitopological space $\left(V, \tau_{G}, \tau_{I G}\right)$ of a graph $G=(V, E)$ are investigated in this part.

Proposition 3.16. Let $\left(\mathrm{V}, \tau_{\mathrm{G}}, \tau_{\mathrm{IG}}\right)$ be the bitopological space of a connected graph $\mathrm{G}=(\mathrm{V}, \mathrm{E})$. Then the set $\mathrm{M}=\{v \in \mathrm{V} \mid \mathrm{d}(v)>1\}$ is $(1,2)$-dense and $(2,1)$-dense in $\mathrm{V}$. 
Proof. From [4], $M$ is dense in $\left(V, \tau_{G}\right)$ of a connected graph $G$ which is not a star. Also by Proposition 2.9, $M$ is dense in $\left(V, \tau_{I G}\right)$ if $G$ is a connected graph. Therefore, $M$ is $(1,2)$-dense in $V$ in a connected graph $\mathrm{G}=(\mathrm{V}, \mathrm{E})$ since $\tau_{\mathrm{IG}}(\mathrm{cl}(\mathrm{M}))=\mathrm{V}$ and then $\tau_{\mathrm{G}}(\mathrm{cl}(\mathrm{V}))=\mathrm{V}$. Similarly $M$ is $(2,1)$-dense in $V$ in a connected graph $\mathrm{G}=(\mathrm{V}, \mathrm{E})$ because the star graph has only one vertex $v \in \mathrm{V}$ such that $\mathrm{d}(v)>1$, then $\tau_{\mathrm{G}}(\mathrm{cl}\{\mathrm{V}\})=\{v\}$ since $\mathrm{V} \backslash\{v\} \in \tau_{\mathrm{G}}$. As a result, $\tau_{\mathrm{IG}}(\mathrm{cl}\{\mathrm{V}\})=\mathrm{V}$ because any non-empty open set in $\tau_{\mathrm{IG}}$ contains $\nu$.

Proposition 3.17. Let $\mathrm{G}=(\mathrm{V}, \mathrm{E})$ be a connected graph which is not a star. Then any non-empty subset of $\mathrm{B}$ is nowhere dense in $\left(\mathrm{V}, \tau_{\mathrm{G}}\right)$ such that $\mathrm{B}=\{v \in \mathrm{V} \mid \mathrm{d}(v)<2\}$.

Proof. Let $\mathrm{N}$ be a non-empty subset of $\mathrm{B}$. It is obvious that $\mathrm{B}=\bigcup_{v \in \mathrm{B}}\{v\}$ and thus $\overline{\mathrm{B}}=\overline{\bigcup_{v \in B}\{v\}}=\bigcup_{v \in \mathrm{B}} \overline{\{v\}}$ by properties of closure (see [6]). Suppose that $u \in \bar{B}$. Thus $u \in \overline{\{v\}}$ for some $v \in B$. From [4], $d(u) \leqslant d(v)=1$. Therefore, $d(u)=1$ and $u \in B$. As a result $\bar{B} \subseteq B$ and so $B$ is closed. This means $c l(B)=B$. Hence for any non-empty subset $\mathrm{N}$ of $\mathrm{B}, \mathrm{cl}(\mathrm{N}) \subseteq \mathrm{cl}(\mathrm{B})=\mathrm{B}$ (see [6]). Now to prove that $\tau_{\mathrm{G}} \mathrm{cl}(\mathrm{N})$ contains no non-empty $\tau_{\mathrm{G}}$ open set. Since $\mathrm{G}$ is not a star, $\mathrm{B} \notin \tau_{\mathrm{G}}$ and so $\tau_{\mathrm{G}} \operatorname{Int}\left(\tau_{\mathrm{G}} \mathrm{cl}(\mathrm{N})\right) \neq B$. For any $\mathrm{F} \subset \mathrm{B}$, we have $A_{u} \neq F$ and $A_{u} \cap A_{r} \neq \mathrm{F}$ for all $u, r \in V$ because $G$ is a connected graph. For this reason, $F$ cannot be written as a union of finitely intersection of elements of $S_{G}$, i.e., $F \notin \tau_{G}$. Hence $\tau_{G} \operatorname{Int}\left(\tau_{G} \operatorname{cl}(N)\right)=\phi \operatorname{since} \operatorname{cl}(N) \subseteq B$ and this means $\mathrm{N}$ is nowhere dense in $\left(\mathrm{V}, \tau_{\mathrm{G}}\right)$.

Proposition 3.18. Let $\mathrm{G}=(\mathrm{V}, \mathrm{E})$ be a connected graph and $\mathrm{n}(\mathrm{V})>2$. Then any non-empty subset of $\mathrm{B}$ is nowhere dense in $\left(\mathrm{V}, \tau_{\mathrm{IG}}\right)$ such that $\mathrm{B}=\{v \in \mathrm{V} \mid \mathrm{d}(v)<2\}$.

Proof. Let $\mathrm{N}$ be a non-empty subset of B. By Proposition 2.8, B is closed set in $\tau_{\mathrm{IG}}$. This means $\mathrm{cl}(\mathrm{B})=\mathrm{B}$. As a result for any non-empty subset $\mathrm{N}$ of $\mathrm{B}, \mathrm{cl}(\mathrm{N}) \subseteq \mathrm{cl}(\mathrm{B})=\mathrm{B}$ (see [6]). Now, to prove that $\tau_{\mathrm{IG}} \mathrm{Cl}(\mathrm{N})$ contains no non-empty $\tau_{I G}$ open set. For any $F \subseteq B$, we have $I_{e} \neq F$ and $I_{e} \bigcap I_{h} \neq F$ for all $e, h \in E$ since $\mathrm{G}$ is a connected graph. As a result, $\mathrm{F}$ cannot be written as a union of finitely intersection of elements of $S_{I G}$, i.e $F \notin \tau_{I G}$. Hence $\tau_{I G} \operatorname{Int}\left(\tau_{I G} \mathrm{cl}(N)\right)=\phi$ since $\mathrm{cl}(N) \subseteq B$ and this means $N$ is nowhere dense in $\left(\mathrm{V}, \tau_{\mathrm{IG}}\right)$.

Corollary 3.19. Let Let $\left(\mathrm{V}, \tau_{\mathrm{G}}, \tau_{\mathrm{IG}}\right)$ be the bitopological space of a connected graph $\mathrm{G}=(\mathrm{V}, \mathrm{E})$ which is not a star and $\mathrm{n}(\mathrm{V})>2$. Then any non-empty subset $\mathrm{N}$ of $\mathrm{B}=\{v \in \mathrm{V} \mid \mathrm{d}(v)<2\}$ is (1,2)-nowhere dense and (2,1)-nowhere dense in $\mathrm{V}(\mathrm{G})$.

Proof. By Propositions 3.17 and 3.18, any non-empty subset $\mathrm{N}$ of $\mathrm{B}=\{v \in \mathrm{V} \mid \mathrm{d}(v)<2\}$ is $(1,2)$-nowhere dense in $V(G)$ since $\tau_{I G} \mathrm{Cl}(N) \subseteq B$ and then $\tau_{G} \operatorname{Int}\left(\tau_{I G} \mathrm{cl}(N)\right)=\phi$. Similarly $N$ is $(2,1)$-nowhere dense in $\mathrm{V}(\mathrm{G})$ because $\tau_{\mathrm{G}} \mathrm{Cl}(\mathrm{N}) \subseteq \mathrm{B}$ and so $\tau_{\mathrm{IG}} \operatorname{Int}\left(\tau_{\mathrm{G}} \operatorname{ciN}\right)=\phi$.

Remark 3.20. If $G$ in Corollary 3.19 is a star, then the non-empty subset $N$ of $B=\{v \in V \mid d(v)<2\}$ is $(2,1)$ nowhere dense in $\mathrm{V}(\mathrm{G})$ since $\tau_{\mathrm{G}} \mathrm{cl}(\mathrm{N})=\mathrm{B}$ for all $\mathrm{N}$ and then $\tau_{\mathrm{IG}} \operatorname{Int}(\mathrm{B})=\phi$. Whereas, only non-empty proper subset $\mathrm{N}$ of $\mathrm{B}=\{v \in \mathrm{V} \mid \mathrm{d}(v)<2\}$ is $(1,2)$-nowhere dense in $\mathrm{V}(\mathrm{G})$ because $\tau_{\mathrm{IG}} \mathrm{cl}(\mathrm{B})=\mathrm{B}$ and so $\tau_{\mathrm{G}} \operatorname{Int}(\mathrm{B})=\mathrm{B} \neq \phi$.

\section{Conclusion}

In this paper a synthesis between graph theory and topology has been made. A bitopological space with every locally finite graph has been associated. Then some properties of this bitopological space have been studied in details. Furthermore, a fundamental step toward studying some properties of locally finite graphs by their corresponding bitopological spaces has been displayed.

\section{References}

[1] E. V. Baby Girija, R. Pilakkat, Bitopological spaces associated with digraphs, South Asian J. Math., 3 (2013), 56-66. 1

[2] A. Bretto, Digital topologies on graphs, Springer-Verlag, Berlin, (2007). 1

[3] B. P. Dvalishvili, Bitopological spaces: theory, relations with generalized algebraic structures, and applications, Elsevier, Amesterdam, (2005). 2

[4] S. M. Jafarian Amiri, A. Jafarzadeh, H. Khatibzadeh, An Alexandroff topology on graphs, Bull. Iranian Math. Soc., 39 (2013), 647-662.1, 3.2, 3.1, 3.1, 3.1, 3.9, 3.3, 3.3 
[5] A. Kilicman, K. Abdulkalek, Topological spaces associated with simple graphs, (submitted). 1, 2

[6] J. M. Moller, General topology, Matematisk Institut, Kobenhavn, (2007). 1, 2, 3.3, 3.3

[7] M. J. Saegrove, On bitopological spaces, Thesis (Ph.D.), Iowa State University, ProQuest LLC, (1971). 1, $2,3.1$

[8] S. Saha Ray, Graph theory with algorithms and its applications, Springer Publishers, New Delhi, (2013). 1, 2

[9] C. Vasudev, Graph theory with applications, New age international, New Delhi, (2006). 1, 2 